\title{
Modalisation of a circularly polarized directive antenna with parasitic elements for wide band applications
}

\author{
Jamil Fouany $^{1}$, Wasim rammal ${ }^{2}$, Mouhamad taoubi ${ }^{2}$, Jamal Rammal $^{1}$ \\ ${ }^{1}$ MUC University, School of Technology, Lebanon \\ ${ }^{2}$ Lebanese University, Faculty of Sciences, Lebanon
}

\begin{abstract}
This paper present a directive circularly polarized wide band antenna with parasitic elements used for a wide band applications. To enhance the directivity and antenna band a distribution of parasitic dipoles is joined to a logarithmic spiral antenna to enhance the forward circular radiation over a bandwidth more than $20 \%$. The electromagnetic responses of the parasitic dipoles are optimized thanks reactive loads welded on to the dipoles antennas.
\end{abstract}

Key words: wide band, parasitic elements, circular polarization, logarithmic spiral.

\section{INTRODUCTION}

With the increasing demands for high quality wireless communication system, circularly polarized antenna have attracted significant attention, since they can avoid the fadings caused by the multiple reflections which can occur along the multipath radio channels [1].Moreover the wideband antennas have received considerable attention to meet the current demand for a wide variety of applications, e.g. electromagnetic compatibility (EMC) measurement, radar [2], and modern communication and sensor systems. The achievement of a circular polarization over a wide bandwidth is a true challenge. Helical and Quadrifilar helix antennas are known to produce circular polarization over a wide bandwidth [3]. However, such traveling wave type antennas must be large in dimension and have a relative high cost. The parasitic element antennas can sometime replace the usual array antennas. This kind of antennas is simpler to achieve because it does not require a feeding network. In such antenna system only one element is fed while the others parasitic ones are excited by the mutual coupling.

The cross YAGI antenna [4] provides a directive beam with circular polarization. This antenna consists of two horizontally and vertically polarized YAGI antennas, phased by $90^{\circ}$ in order to create a circular polarization. These antennas have two feeding elements who manage independently the two linear polarizations by the parasitic elements. Another category of circularly polarization parasitic antenna has also been developed in printed versions of patch type [5].Another architecture of $2 \mathrm{D}^{1 / 2}$ Yagi-Uda antennas which radiates in circular polarization was proposed by YAN [6]in 2013, this work is based on the work of SUN in 2010[6]. This architecture consists of three rectangular stacked loops with shape modification.

The most usual way for designing this kind of antennas uses massive parametric electromagnetic simulation; however other various methods were investigated. For example, a parasitic elements antenna circularly polarized for RFID handheld reader 0 with one active element was designed with a particle swarm optimization of the whole dimensions of the antenna Another parasitic antenna structure was designed thanks to an experimental research of the reactive circuits that must be connected to the parasitic elements in order to realize a desired radiation pattern Error! Reference source not found.

In this paper we investigate a new wide band antenna architecture which is a parasitic elements antenna optimized in order to achieve both a directivity enhancement and a circular polarization. The architecture associates a printed logarithmic spiral antenna [8]with a 3D distribution of parasitic dipoles. A logarithmic spiral offers a bidirectional radiation (backward and forward radiation). One lobe is right hand circularly polarized and the second is left hand polarized. The parasitic dipoles are set to synthesize a reflector and a director able simultaneously to eliminate the backward radiation and to focus the forward beam while maintaining its circular polarization. The parasitic dipoles are the degrees of freedom which enable the optimization the performances of the antenna system. This work declines the synthesis method described in to develop the wide band circularly polarized directive antenna with parasitic elements. The synthesis method determines the reactive loads which provide the properties that comply with the desired radiation pattern. In this paper the synthesis results are compared to full-wave simulations.

\section{SYNTHESIS METHOD PROCEDURE}

To find the reactive loads that must be welded onto the parasitic printed dipoles the antenna is first simulated as a multiport system. One port is affected to each parasitic dipole at the location where the SMD loads must be connected. This Full wave simulation provides the scattering matrix of the whole antenna system and the radiation patterns of each antenna (or parasitic element). The synthesis determines the optimal reactive loads which achieve the desired radiation 
Jamil Fouany et al., International Journal of Microwaves Applications, 7(2), March - April 2018, 19 - 23

objective. The synthesis procedure solves $(1,2)$. In (1) the reactive loads are changed into the reflection coefficients $\Gamma i$. the $[\Gamma]$ matrix is diagonal. $[\mathrm{S}]$ Is the scattering matrix of the antenna system. (a) Is a vector containing the normalized power waves applied to the antenna ports. These weights depend on the antenna S matrix and the reactive loads that will be connected to the parasitic elements (Fig1). The radiation pattern $\Phi$ of the array is a linear combination of the elementary radiation patterns $\Phi$ i of each elementary antenna.

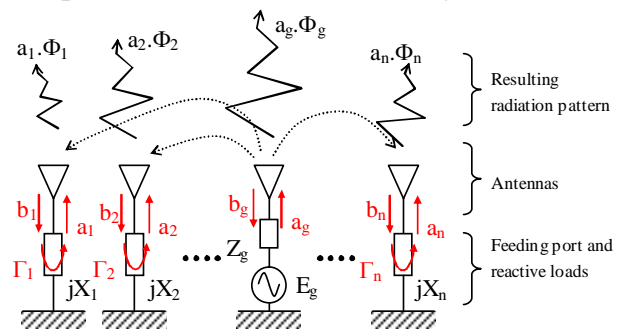

Figure 1: Principle of a parasitic antenna array

$$
\begin{aligned}
& {\left[\mathrm{I}-[\Gamma][[\mathrm{S}]] .(\mathrm{a})=\left(\mathrm{a}_{0}\right)\right.} \\
& \overrightarrow{\Phi(\theta, \varphi)}=\left[\begin{array}{lllll}
\overrightarrow{\Phi_{1}} & \ldots & \overrightarrow{\Phi_{\mathrm{g}}} & \ldots & \overrightarrow{\Phi_{\mathrm{n}}}
\end{array}\right]\left(\begin{array}{c}
\mathrm{a}_{1} \\
\vdots \\
\mathrm{ag}_{\mathrm{g}} \\
\vdots \\
\mathrm{a}_{\mathrm{n}}
\end{array}\right)
\end{aligned}
$$

The electric fields vectors contain into each elementary radiation pattern allow the optimization on the radiated polarization. For more details about the method readers can refer to [7].

\section{DESCRIPTION OF THE ANTENNA}

In this paper, a parasitic antenna operating at $2.45 \mathrm{GHz}$ has been studied. This is a typical antenna with parasitic elements. In this case, the reactive loads $\mathrm{Xi}$ are calculated and implanted directly into the final design of the antenna. This technology has already been validated for linearly polarized antennas [8]. The analytical synthesis allows achieving the optimum performances in terms of radiation pattern, directivity and polarization. The global architecture of the antenna system is presented on Fig.2. This architecture consists in a logarithmic spiral antenna (driven element) located between two layers of printed parasitic dipoles operating as reflectors or directors. Each layer is composed of a set of 4 dipoles printed on a FR4 substrates $(\varepsilon r=4.1$, loss tangent $=0.04)$. The spacing between the substrates is $0.3 \lambda$. All parasitic dipoles are $1 \mathrm{~mm}$ width and $41.8 \mathrm{~mm}$ length.

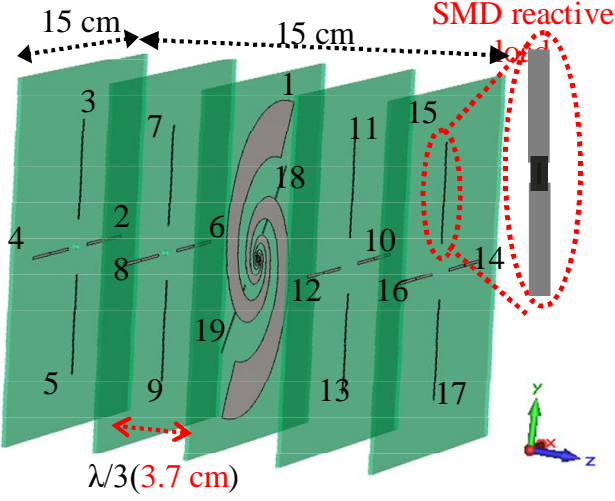

Figure 2: Design of the proposed antenna

Since the spiral isn't $90^{\circ}$ symmetric, two extra dipoles are printed on the back face of the spiral as shown in Fig.3.These two extra tuning dipoles offer the extra degree of freedom useful to balance the amplitudes of the field's vectors coupled along the two orthogonal directions $\mathrm{x}$ and $\mathrm{y}$.

Fig. 4 shows the feeding of the antenna. It is composed of a 9 $\mathrm{cm}$ length miniature coaxial cable, shielded with a $3 \mathrm{~cm}$ length copper tube $(\lambda / 4)$. The latter provides a balanced excitation for the spiral antenna

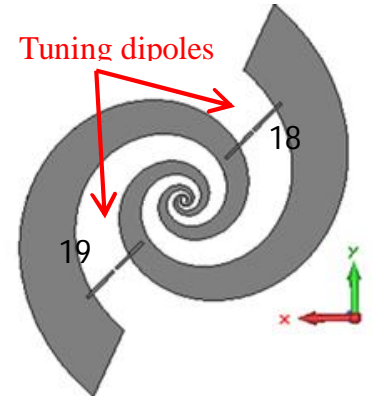

Figure 3: Position of the equilibrating dipoles on the rear face of the substrate supporting the logarithmic spiral antenna

To perform the full-wave simulation, 19 numerical discrete ports ( 1 for the spiral and 18 for the parasitic dipoles) are required. The matrix $[\mathrm{S}]$ and the 19 elementary radiation patterns of the global antenna system are extracted. The synthesis search the best set of reactive loads which tunes the phase and equals the magnitude of the radiated fields. Simultaneously, the synthesis optimizes the forward directivity. The values of the synthetized reactive loads are presented in the table 1 . 
Jamil Fouany et al., International Journal of Microwaves Applications, 7(2), March - April 2018, 19 - 23

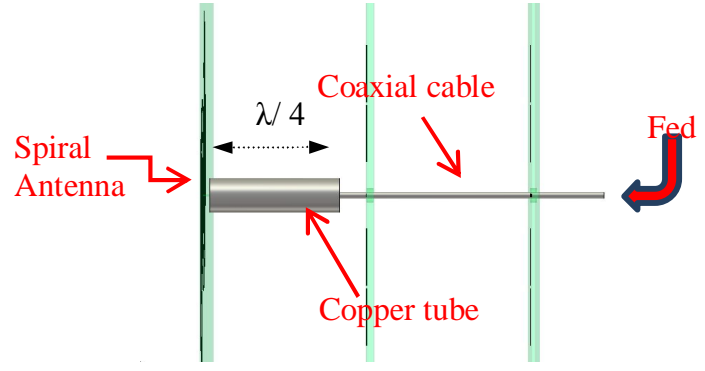

Figure 4: Feeding of the antenna

Table 1: The values of synthesis reactive loads

\begin{tabular}{|c|c|c|}
\hline dipoles & $\begin{array}{c}\text { Inductance } \\
(\mathrm{nH})\end{array}$ & $\begin{array}{c}\text { Capacities } \\
(\mathrm{pF})\end{array}$ \\
\hline 2 and 4 & 1.6 & \\
\hline 3 and 5 & 1.3 & 6.3 \\
\hline 6 and 8 & & 3.4 \\
\hline 7 and 9 & & 0.2 \\
\hline 10 and 12 & & 0.3 \\
\hline 11 and 13 & & 0.5 \\
\hline $14,15,16$ and 17 & & \\
\hline 18 and 19 & 18 & \\
\hline
\end{tabular}

In order to validate these values a global full-wave simulation with reactive lumped elements is achieved. The next paragraph compares the synthesis results with the CST full-wave simulation including the lumped loads.

\section{SIMULATION OF THE ANTENNA DESIGN}

In this partwe present comparison between simulation and synthesis calculus of the global antenna (19 elements) and we justify the importance of the 2 tuning dipoles to optimize the axial ratio.

\subsection{Performances of the spiral antenna alone (without the parasitic elements)}

The frequency evaluation of axial ratio and directivity of the spiral antenna alone, without the parasitic elements is shown in figure 5.A realized gain of $4.3 \mathrm{dBis}$ obtained at $2.45 \mathrm{GHz}$ and decreases slightly out of the operatingfrequency, whereas the minimum of axial ratio obtained is a $1.8 \mathrm{~dB}$ at the operating frequency.

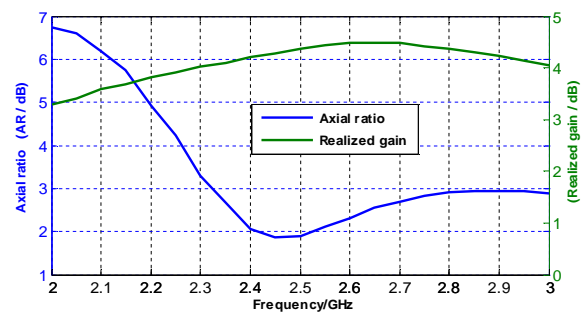

Figure 5: Frequency evolution of the realized gain and the axial ratio of the spiral antenna alone

\subsection{Performances of the global antenna (19 elements)}

In this part we present a comparison between the full wave simulation and the synthesis calculus. We compare a CST full wave simulation including the lumped capacities and inductors connected to the parasitic dipoles. The synthesis and simulated axial ratio in the $\phi=0^{\circ}$ cut plane at $2.45 \mathrm{GHz}$ is shown inFig.6. A synthesis value of $0.53 \mathrm{~dB}$ is obtained in the broad side and remains lower than $3 \mathrm{~dB}$ for an aperture angle of $\pm 20^{\circ}$.Fig. 7 show the synthesis and simulated radiation pattern in the 2 cut planes $\phi=0^{\circ}$.A $11 \mathrm{~dB}$ directivity is obtained at broad side and the backward directivity is about$9 \mathrm{~dB}$. As expected, these figures show asymmetrical radiation pattern. The return loss predicted through the synthesis is quite similar to the one computed by the full-wave electromagnetic simulation. The comparison is shown in Figure 8. The return loss is lower than $-10 \mathrm{~dB}$ from $2 \mathrm{GHz}$ to $2.7 \mathrm{GHz}$.

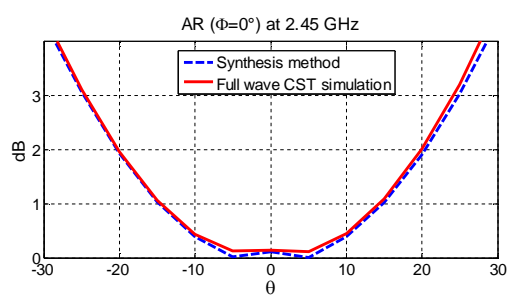

Figure 6: Comparaison of the $\mathrm{AR}$ in the phi $=0^{\circ}$ cut planepredicted by the synthesis method

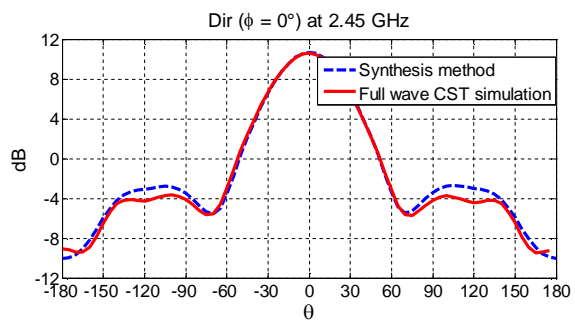

Figure 7: Comparaison of the directivity in the phi $=0^{\circ}$ cut planepredicted by the synthesis methodwith the full wave simulation. 
Jamil Fouany et al., International Journal of Microwaves Applications, 7(2), March - April 2018, 19 - 23

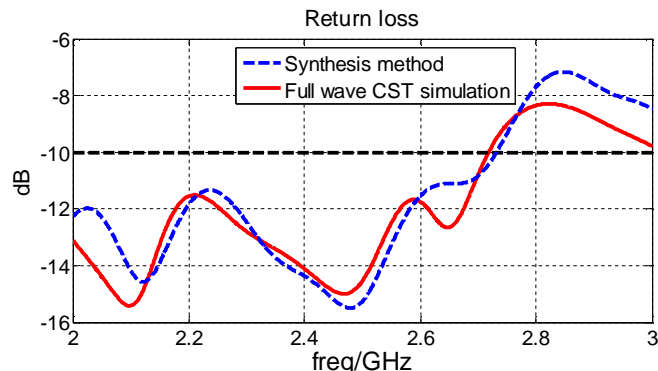

Figure 8: Simulated and synthesis return loss (S11 dB)

All these comparisons validate the design process. The parasitic element antenna that is proposed in this paper offers an axial ratio is lower than $3 \mathrm{~dB}$ and a $\mathrm{S} 11$ is better than $-10 \mathrm{~dB}$ over this bandwidth.

\subsection{Assessment of the tuning dipoles effect}

Since the synthesis method is based on the phase optimization, two tuning dipoles are used to balance the amplitudes of the field's vectors coupled by the driven antenna and the others parasitic elements. A comparison between the synthesis results of the antenna with and without tuning dipoles will be presented. The simulated axial ratio (AR) of the antenna in the phi $=0^{\circ}$ plane at $2.45 \mathrm{GHz}$ is shown in Fig.9. A $1.2 \mathrm{~dB}$ AR is obtained at $\theta=0^{\circ}$ and it stays lower than $3 \mathrm{~dB}$ for an aperture angle $\theta= \pm 20^{\circ}$. A $1 \mathrm{~dB}$ improvement is obtained with the tuning dipoles. The frequency dependence of the axial ratio is shown in Fig.12. Without the tuning dipoles the axial ratio is lower than $3 \mathrm{~dB}$ from 2.15 to $2.55 \mathrm{GHz}(400 \mathrm{MHz})$ and strongly increases for upper frequencies. It can be observed that with the tuning dipoles the AR stays lower $3 \mathrm{~dB}$ from 2.2 to $2.7 \mathrm{GHz}(500 \mathrm{MHz})$ and it is contained lower $4 \mathrm{~dB}$ over an $800 \mathrm{MHz}$ of band. The directivity is always greater than $7.6 \mathrm{~dB}$ over the entire band with a maximum of $10.6 \mathrm{~dB}$ at $2.45 \mathrm{GHz}$ (Fig10).

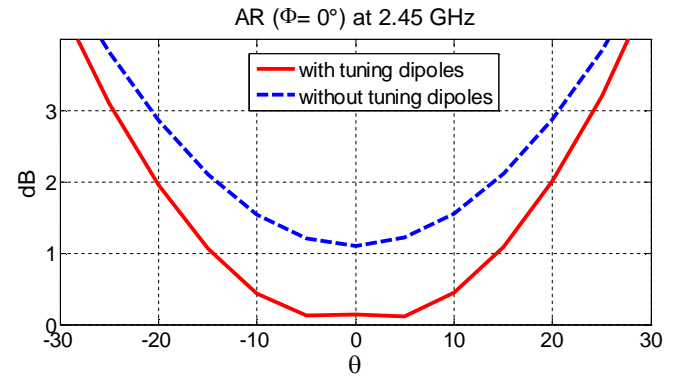

Figure 9: Comparaison of the $\mathrm{AR}$ of the antenna at $2.45 \mathrm{GHz}$ with and without the tuning dipoles

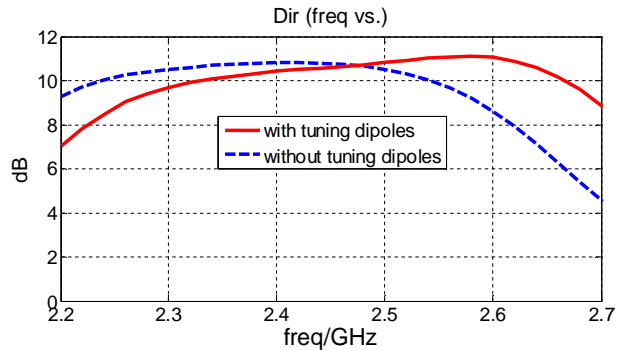

Figure 10: Frequency evolution of directivity with and without the tuning dipoles

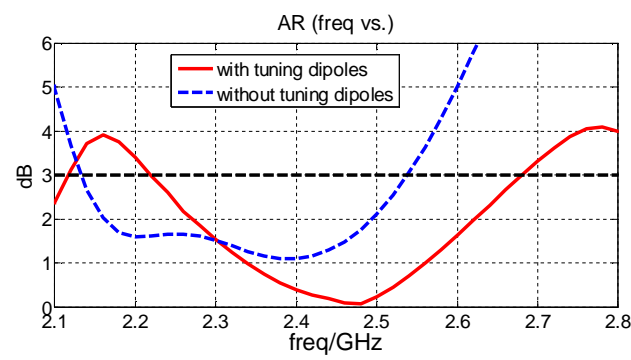

Figure 11: Frequency evolution of $A R$ with and without the tuning dipoles

\section{CONCLUSION}

The design technique leads to an original concept. This original design associates a spatial distribution of parasitic dipoles with a spiral antenna. These dipoles are loaded with lumped inductors and capacitors. The new antenna system improves the directivity of the spiral antenna from $4.3 \mathrm{~dB}$ to 11 $\mathrm{dB}$. The axial ratio is lower than $3 \mathrm{~dB}$ over a $20 \%$. Relative frequency bandwidth. The prototype was fabricated and measured to verify the simulations. In this design the reactive functions are realized by SMD components. The measured results showed good agreement with the simulations.

\section{REFERENCES}

[1] D.S. Lerner, A wave polarization converter for circular polarization, IEEE Trans Antennas Propag AP-13 (January 1965), 3-7. https://doi.org/10.1109/TAP.1965.1138367

[2] F. Karshenas, A. R. Mallahzadeh, and A. Imani Modified TEM Horn Antenna for Wideband Applications, 2009 13th International Symposium on Antenna Technology and Applied Electromagnetics and the Canadian Radio Sciences Meeting. https://doi.org/10.1109/ANTEMURSI.2009.4805077

[3] D. F. Filipovic, M. A. Tassoudji, and E. Ozaki, A coupled-segmentquadrifilar helical antenna, In Proc. IEEE MTT-S Symp. TechnologiesWireless Applications Dig., Feb. 1997, pp. 43-46.

[4] Louis D.Breetz, A Circularly Polarized Yagi Antenna System for NTS-I and NTS-2 Ground Stations, Naval Research Laboratory; Sep 1976.

[5] Johan Huang, Planar Microstrip Yagi antenna array, Patent Number 5220335, united stat, 15, Jun, 1993. 
Jamil Fouany et al., International Journal of Microwaves Applications, 7(2), March - April 2018, 19 - 23

[6] Yan Li, Jun Ouyang, and Peng Yang, A circularly polarized compact Ántenna for UHF band RFID reader," Progress In Electromagnetic Research Letters, Vol. 42, 119\{127, 2013\}.

[7] Sun, S. and L. Zhu, Miniaturized patch hybrid coupler using asymmetrically loaded cross slots, IET Microwav Antennas Propagation, Vol. 4, No. 9, 1427\{1433, Sep. 2010

[8] J. Thaysen, K. B. Jakobsen, and J. Appel-Hansen, A logarithmic spiral antenna for 0.4 to $3.8 \mathrm{GHz}$. Applied Microwave \& Wireless, pp. 32-45, Feb. 2001. 